\title{
Intraosseous basivertebral nerve ablation for the treatment of chronic low back pain: a prospective randomized double-blind sham-controlled multi-center study
}

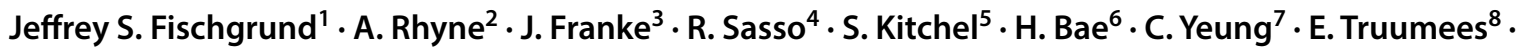

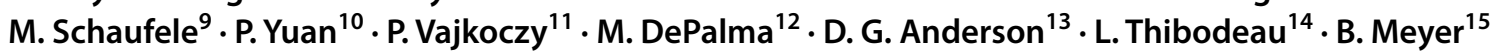

Received: 5 October 2017 / Revised: 11 January 2018 / Accepted: 24 January 2018 / Published online: 8 February 2018

(c) The Author(s) 2018. This article is an open access publication

\begin{abstract}
Purpose To evaluate the safety and efficacy of radiofrequency (RF) ablation of the basivertebral nerve (BVN) for the treatment of chronic low back pain (CLBP) in a Food and Drug Administration approved Investigational Device Exemption trial. The BVN has been shown to innervate endplate nociceptors which are thought to be a source of CLBP.

Methods A total of 225 patients diagnosed with CLBP were randomized to either a sham (78 patients) or treatment (147 patients) intervention. The mean age within the study was 47 years (range 25-69) and the mean baseline ODI was 42 . All patients had Type I or Type II Modic changes of the treated vertebral bodies. Patients were evaluated preoperatively, and at 2 weeks, 6 weeks and 3, 6 and 12 months postoperatively. The primary endpoint was the comparative change in ODI from baseline to 3 months.

Results At 3 months, the average ODI in the treatment arm decreased 20.5 points, as compared to a 15.2 point decrease in the sham arm $(p=0.019$, per-protocol population). A responder analysis based on ODI decrease $\geq 10$ points showed that $75.6 \%$ of patients in the treatment arm as compared to $55.3 \%$ in the sham control arm exhibited a clinically meaningful improvement at 3 months.

Conclusion Patients treated with RF ablation of the BVN for CLBP exhibited significantly greater improvement in ODI at 3 months and a higher responder rate than sham treated controls. BVN ablation represents a potential minimally invasive treatment for the relief of chronic low back pain.
\end{abstract}

Graphical abstract These slides can be retrieved under Electronic Supplementary Material.
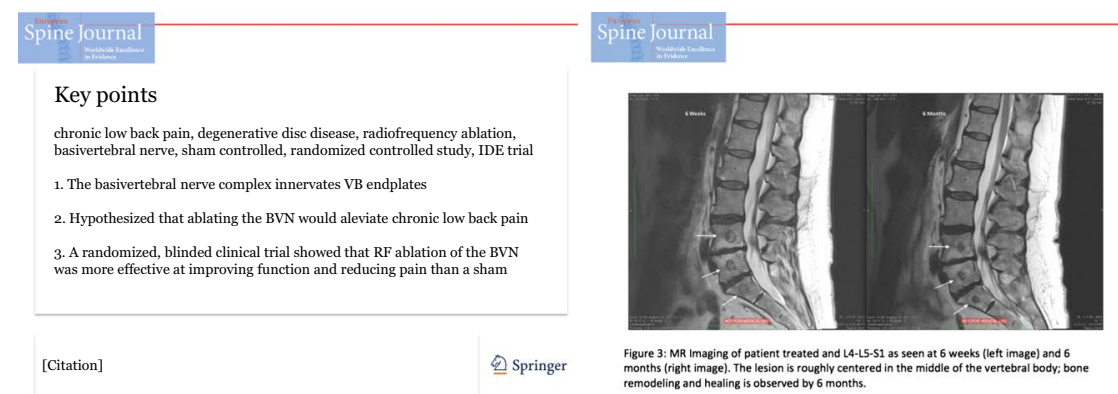

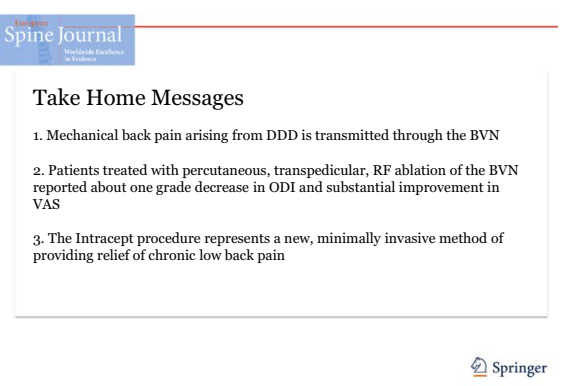

Electronic supplementary material The online version of this article (https://doi.org/10.1007/s00586-018-5496-1) contains supplementary material, which is available to authorized users.

Extended author information available on the last page of the article 
Keywords Chronic low back pain $\cdot$ Degenerative disc disease $\cdot$ Radiofrequency ablation $\cdot$ Basivertebral nerve $\cdot$ Sham controlled $\cdot$ Randomized controlled study $\cdot$ IDE trial

\section{Introduction}

Low back pain (LBP) is the most common cause of activity limitation in individuals younger than 45 years of age. While conservative and invasive treatments may be used to treat CLBP, both may prove to be only moderately effective and/or of temporary duration. For example, Fritzell et al. found an 11 point decrease in ODI in patients fused for CLBP, but also noted that the improvement in pain gradually deteriorated after 6 months [1]. Brox et al. suggested that the success rate of fusion was little better than cognitive intervention [2]. Although several authors have shown good improvement in outcome measures following total disc replacement and/or lumbar fusion [3, 4], both are invasive surgical interventions with prolonged recovery periods [5].

Chronic low back pain has been associated with disc degeneration and discogenic pain, attributed to pain signals arising from nerves in the posterior annulus. In addition, the existence and role of nerves within bone as well as within the disc is documented. For example, Sherman described the rich nerve supply in human bones, including within the vertebral body, and noted that these nerves mostly appeared to be non-myelinated [6].

Crock and Yoshizawa mapped the vertebral body vessels and reported that the nerves were collocated with the vessels [7]. In 1997, Brown et al. studied the cartilage endplates and underlying cancellous bone obtained from patients operated for degenerative disc disease (DDD) presenting with and without chronic back pain. In patients with severe back pain, they observed proliferation of blood vessels at the disc-endplate interface, which they identified as sensory nerves using immunohistochemical techniques including immunoreactivity to substance P. They noted that the increased density of nociceptors in conjunction with endplate cartilage defects strongly suggested that the endplates and vertebral bodies were the sources of pain in patients with DDD [8].

Specific mapping of human vertebral body nerves was performed by Antonacci et al. who examined 69 vertebral bodies and found that nerves entered the vertebral body posteriorly via the basivertebral foramen. These basivertebral nerves, which branch from the sinuvertebral nerve, followed the course of the nutrient arteries, clustering at the vertebral center, then branching to the endplates. They postulated that these nerves could play a role in low back pain [9]. Similarly, Bailey et al. used PGP 9.5 staining to describe the morphology of the BVN including its vertebral body entry through posterior foramina [10]. Confirmatory evidence that the BVN complex transmits nociceptive signals was made by
Fras et al. who observed the basivertebral nerves stained for the presence of substance $P$ [11].

While sensitized nociceptors within the posterior annulus of degenerating discs have been identified as a source or origin for so called "discogenic" low back pain, the presence, anatomic course and nociceptive capacity of the BVN suggests an additional hypothesis: that in some patients the origin of low back pain is the vertebral endplates, and that pain is transmitted through signals emanating in the BVN complex. This hypothesis is buttressed by the observation that the density of endplate and vertebral body innervation via the basivertebral nerve is higher than that of the annulus, suggesting that some CLBP traditionally reported as discogenic in origin is in fact vertebrogenic [12]. Clinical evidence into the role of the BVN in the transmission of pain in patients with CLBP was provided by Becker et al. They used unilateral transpedicular access and RF energy to ablate the BVN in a series of 16 patients presenting with axial back pain, who were subsequently followed for 1 year. In their series, they observed an improvement in ODI from $52 \pm 13$ at baseline to $23 \pm 21$ at 3 months $(p<0.001)$; the improvement was maintained out to 1 year [13].

We report on a double-blind, randomized, clinical trial designed to further test the hypothesis that interrupting pain transmission from the BVN could alleviate CLBP in patients with Modic type 1 or 2 abnormalities of the endplates.

\section{Methods}

\section{Study design}

Between October 2011 and February 2014, 225 patients were randomized to a prospective, multi-center, doubleblind, sham-controlled, FDA approved Investigational Device Exemption clinical trial (registered with ClinicalTrials.gov as NCT01446419) at 15 investigational sites in the United States and three sites in Germany. The primary objective was to evaluate the safety and efficacy of using RF energy to ablate the BVN for the treatment of chronic axial low back pain.

The primary inclusion criteria included skeletally mature patients with chronic ( $\geq 6$ months), isolated lumbar pain, who had not responded to at least 6 months of non-operative management. Type 1 or Type 2 Modic changes were required at the proposed treatment levels and treatment was limited to a minimum of two and a maximum of three consecutive vertebral body levels from L3-S1. In addition, candidate 
patients had to report a minimum ODI of 30 points (100 point scale) and a minimum VAS of $4 \mathrm{~cm}(10 \mathrm{~cm}$ scale).

Exclusion criteria included radicular pain, previous lumbar spine surgery, symptomatic spinal stenosis, diagnosed osteoporosis $(T<2.5)$, disc extrusion or protrusion $>5 \mathrm{~mm}$, spondylolisthesis $>2 \mathrm{~mm}$ at any level, 3 or more Waddell's signs of Inorganic Behavior, and a Beck Depression Inventory of greater than 24 . Radicular pain was considered to be any pain that traveled along a dermatomal distribution into the lower extremity, causing pain, numbness, and/or weakness/heaviness of the affected area. Symptomatic spinal stenosis was defined as the presence of neurogenic claudication as confirmed by imaging, with symptoms typically including a combination of discomfort, pain, numbness, and weakness in the calves, buttocks, and/or thighs, often brought on by walking or prolonged standing, but relieved by flexion or sitting. In addition, patients involved in litigation related to back pain or injury, patients receiving disability compensation, and patients prescribed extended release narcotics were not eligible.

Final selection of study patients was arbitrated by a panel of three spine surgeons of which two had to agree on the proposed patient's medical, clinical, and radiographic presentation prior to inclusion in the study.

Once a patient was enrolled, pre-operative mapping was performed at each candidate vertebral body to determine the relative location of the branch point or terminus of the BVN, typically between 40 and $60 \%$ of the posterior to anterior distance across the vertebral body. An example showing the determination of the relative position of the BVN terminus is shown in Fig. 1.

Patients were randomized using 2:1 block randomization to either the treatment or the sham arm. Randomization occurred at each investigational site in random blocks of six or nine patients and arm determination was made after the patient was under anesthesia. Anesthetic choice was a function of patient/physician practices and preferences; 113 of $225(50.2 \%)$ of patients in the study received general anesthesia and 112 of 225 (49.8\%) of patients received moderate conscious sedation.

The treatment arm patients received thermal ablation at the terminus of the BVN using a transpedicular delivery system (Intracept ${ }^{\circledR}$ System, Relievant Medsystems, Redwood City, CA, USA). This system consisted of a set of access instruments, straight and curved cannulae, a bipolar $\mathrm{RF}$ probe to deliver thermal energy, and an RF generator.

The procedure is performed unilaterally with the patient in a prone position; either general or conscious sedation is administered. Using standard anatomic landmarks, the location of the entry pedicle at each level to be treated is determined and marked. Under fluoroscopic guidance, an introducer cannula is advanced through the pedicle until

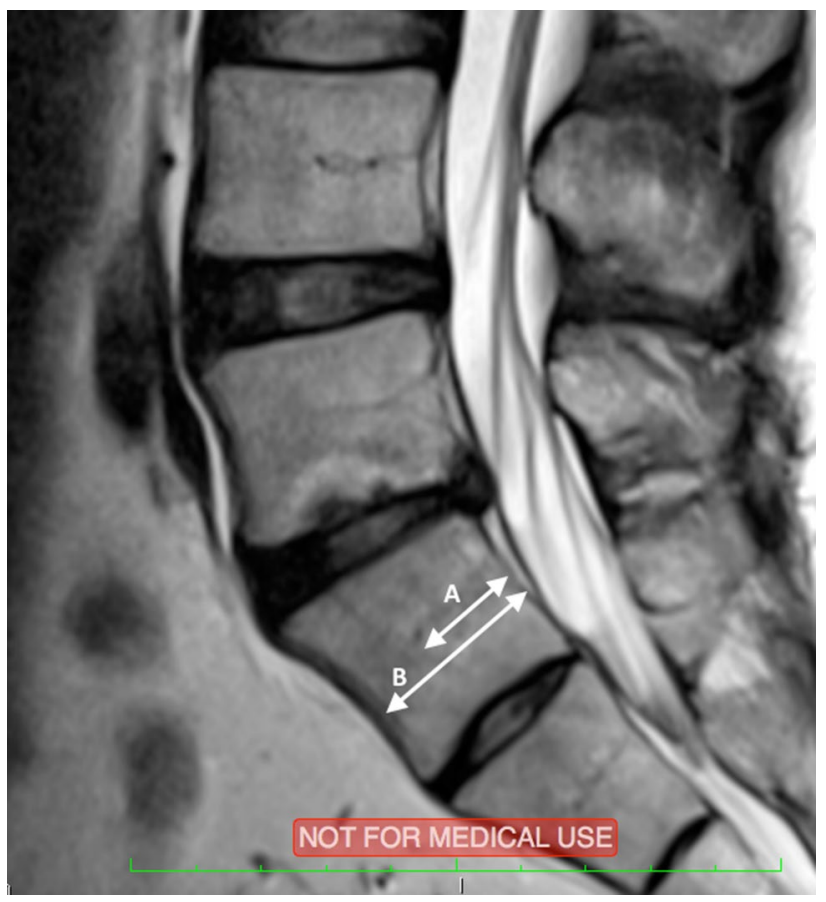

Fig. 1 Targeting of the ablation is performed preoperatively on a sagittal or coronal (not shown) image of the level to be treated. The distance from the posterior wall to the end of the channel with the basivertebral vessels (A) is measured and divided by the overall posterior-to-anterior dimension of the vertebral body (B). The ratio $\mathrm{A} / \mathrm{B}$ is noted and the RF probe is guided to the same relative position under fluoroscopic guidance during the treatment procedure

the trocar just breaches the posterior vertebral wall. The introducer trocar is exchanged with a smaller plastic cannula/curved nitinol stylet assembly, which facilitates the creation of a curved path from the posterior wall to the pre-determined target located at the terminus of the BVN, located near the center of the vertebral body. Finally, the curved nitinol stylet is removed and an RF probe is introduced positioned at the terminus of the BVN. The bipolar $\mathrm{RF}$ probe is activated and the temperature at the tip is maintained at a constant $85{ }^{\circ} \mathrm{C}$ for $15 \mathrm{~min}$, a temperature and time determined in bovine pre-clinical models to create an approximately $1 \mathrm{~cm}$ spherical lesion within the vertebral body.

Patients in the sham arm underwent the same operating room protocol for the same overall duration as the treatment arm patients. However, the sham surgical procedure consisted only of docking the introducer cannula 1-2 mm into the pedicle and simulating the RF ablation, including an equivalent dwell time. To maintain blinding, the treating and follow-up physicians differed. At 1 year, patients in the sham arm were permitted to cross-over to the active treatment. 


\section{Clinical and radiographic outcome measurements}

Outcomes were evaluated using the Oswestry Disability Index questionnaire (ODI) and the Medical Outcomes Trust ShortForm Health Survey (SF-36). Back pain was assessed using a visual analog scale (VAS) on a 10-cm scale ranging from 0 (no pain) to 10 (worst imaginable pain). Physical and neurological examinations were performed at each interval, while MR imaging was performed at 6 weeks and 6 months. MR imaging (T1, T2, and STIR time constants) was obtained at the 6-week and 6-month follow-up visits. Radiographic assessments were performed by a blinded independent radiologist. Patients were assessed for changes in Modic Type presentation at L3 through S1 at 6-week and 6-month follow-up.

\section{Statistical and data evaluation methods}

Sample size was calculated assuming mean ODI would exhibit a standard deviation of 20 points and the study was powered to detect a 10 point between-group difference in ODI with $90 \%$ power and $\alpha=0.05$. These assumptions yielded a sample size of 133 treatment and 67 sham patients.

The primary efficacy endpoint was the 3 month change in ODI. Descriptive statistics evaluated the change using an analysis of covariance, with the covariates consisting of treatment group, analysis center, treatment group by analysis center interaction, and the baseline ODI score. The SF-36 and VAS metrics were analyzed similarly.

Two pre-planned analysis groups were defined within the FDA approved IDE protocol which governed the study. These were the intent-to-treat (ITT) and the per-protocol (PP) populations. The ITT population consisted of the patients as randomized. However, because the study was randomized to a surgical procedure, the possibility of an unsuccessful procedure was admitted, with the most likely cause being failure of the RF generated lesion to be collocated with the terminus of the BVN. The per-protocol population excluded such patients as well as any patients in which the procedure could not be carried out, or who were not compliant with the post-operative protocol.

The safety endpoints consisted of the incidence and severity of adverse events and serious adverse events, both procedure and device related, the maintenance of neurological status, and the radiographic determination of appropriate lesion safety margin in each treated vertebral body.

\section{Results}

\section{Patient demographics}

Two hundred and twenty-five patients were enrolled (147 treatment and 78 sham); demographics and baseline characteristics were similar between groups (Table 1). Follow-up was $98.7,98.2$, and $96.9 \%$ at 3, 6, and 12 months, respectively. Six patients exited the study: two patients withdrew consent prior to evaluation at 3 months; three patients were terminated after choosing to undergo invasive surgical procedures on the spine; one patient died prior to the 12 month visit for reasons not related to the study. See Fig. 2 for study flow diagram by treatment arm.

\section{Treatment success}

All patients were successfully treated except for one treatment arm patient with extremely dense bone at S1. Targeting success was defined as overlap between the RF created ablation zone and the terminus of the BVN at each level treated observed on 6 week MR imaging. Targeting was successful in 129 of 145 patients $(89.0 \%)$ or in 300 of 317 treated vertebral bodies (94.6\%). Lack of targeting success at any one level removed the patient from the ITT group as both the cephalad and caudal vertebral body at an affected level were required to be successfully treated. Representative MR imaging of the lesion created to ablate the BVN at 6 weeks and 6 months is shown in Fig. 3.

\section{Levels treated}

Most commonly L5-S1 (122/224 patients) and L4-L5 (45/224) followed by L4-L5-S1 (41/224) were treated. An additional nine patients were treated only at L3-L4 with seven patients treated at L3-L4-L5.

\section{ODI}

In the ITT population at 3 months, the treatment group exhibited a 19.0 least squares mean (LSM) improvement in ODI compared to a 15.4 LSM improvement in the sham group ( $p=0.107)$. In the PP population at 3 months, the treatment group exhibited a 20.5 LSM improvement in ODI compared to a 15.2 LSM improvement in the sham group $(p=0.019)$. The primary outcome analysis is shown in Table 2.

Using a 10-point improvement in ODI, the commonly accepted minimum clinically important difference (MCID) in the treatment of CLBP $[14,15], 75.6 \%$ of treatment arm patients compared with $55.3 \%$ of sham arm patients exhibited a successful response.

\section{VAS}

The LSM improvement in VAS in the treatment arm was 2.97, 3.04, and $2.84 \mathrm{~cm}$ at 3,6 , and 12 months, respectively. The LSM improvement in VAS in the sham arm was $2.36,2.08$, and $2.08 \mathrm{~cm}$ at 3,6 , and 12 months, respectively 
Table 1 Patient demographics and baseline characteristics

\begin{tabular}{|c|c|c|c|}
\hline Characteristic & Intracept system arm $(n=147)$ & Sham control arm $(n=78)$ & $p$ \\
\hline Age (years), mean (range) & $46.9(26-69)$ & $47.1(25-69)$ & $0.869^{\mathrm{a}}$ \\
\hline Male, $n(\%)$ & $82(55.8 \%)$ & $41(52.6 \%)$ & $0.708^{\mathrm{b}}$ \\
\hline BMI $\left(\mathrm{kg} / \mathrm{m}^{2}\right)$, mean (range) & $27.44(18.9-38.4)$ & $27.16(19.2-38.0)$ & $0.666^{\mathrm{a}}$ \\
\hline Caucasian, $n(\%)$ & $134(91.2 \%)$ & $71(91.0 \%)$ & $0.409^{b}$ \\
\hline Married, $n(\%)$ & $101(68.7 \%)$ & $50(64.1 \%)$ & $0.142^{\mathrm{b}}$ \\
\hline College degree or higher, $n(\%)$ & $87(59.2 \%)$ & $47(60.3 \%)$ & $0.535^{\mathrm{b}}$ \\
\hline Working before procedure, $n(\%)$ & $110(74.8 \%)$ & $57(73.1 \%)$ & $0.328^{\mathrm{b}}$ \\
\hline Current tobacco use, $n(\%)$ & $25(17.0 \%)$ & $10(12.8 \%)$ & \\
\hline \multicolumn{4}{|l|}{ Duration low back symptoms, $n(\%)$} \\
\hline$\geq 6$ months to $<1$ year & $6(4.1 \%)$ & $4(5.1 \%)$ & $0.990^{\mathrm{c}}$ \\
\hline$\geq 1$ year to $<2$ years & $15(10.2 \%)$ & $8(10.3 \%)$ & \\
\hline$\geq 2$ years to $<3$ years & $10(6.8 \%)$ & $5(6.4 \%)$ & \\
\hline$\geq 3$ years to $<5$ years & $18(12.2 \%)$ & $7(9.0 \%)$ & \\
\hline$\geq 5$ years & $98(66.7 \%)$ & $54(69.2 \%)$ & \\
\hline Opioid use before procedure, $n(\%)$ & $51(34.7 \%)$ & $27(34.6 \%)$ & $0.872^{\mathrm{b}}$ \\
\hline \multicolumn{4}{|l|}{ Modic changes, $n(\%)$} \\
\hline Type 1 & $46(31.3 \%)$ & $29(37.2 \%)$ & $0.578^{\mathrm{b}}$ \\
\hline Type 2 & $89(60.5 \%)$ & $42(53.8 \%)$ & \\
\hline Type 1 and Type 2 & $12(8.2 \%)$ & $7(9.0 \%)$ & \\
\hline ODI mean (range) & $42.9(30-76)$ & $41.1(26-78)$ & $0.277^{\mathrm{a}}$ \\
\hline VAS mean (range) & $6.82(4.0-10.0)$ & $6.63(4.0-9.1)$ & $0.343^{\mathrm{a}}$ \\
\hline BDI mean (range) & $7.7(0-23)$ & $7.6(0-24)$ & $0.853^{\mathrm{a}}$ \\
\hline SF-36 PCS mean (range) & $33.22(14.83-48.11)$ & $34.07(14.01-54.15)$ & $0.407^{\mathrm{a}}$ \\
\hline SF-36 MCS mean (range) & $51.97(23.05-69.06)$ & $52.72(20.07-73.38)$ & $0.579^{\mathrm{a}}$ \\
\hline
\end{tabular}

BDI Beck Depression Inventory, PCS physical component summary, MCS mental component summary ${ }^{\mathrm{a}} p$ value from a two-way ANOVA with treatment group and analysis center as factors

${ }^{\mathrm{b}} p$ value from a CMH general association test stratified by analysis center

${ }^{c} p$ value from a CMH row mean scores test stratified by analysis center

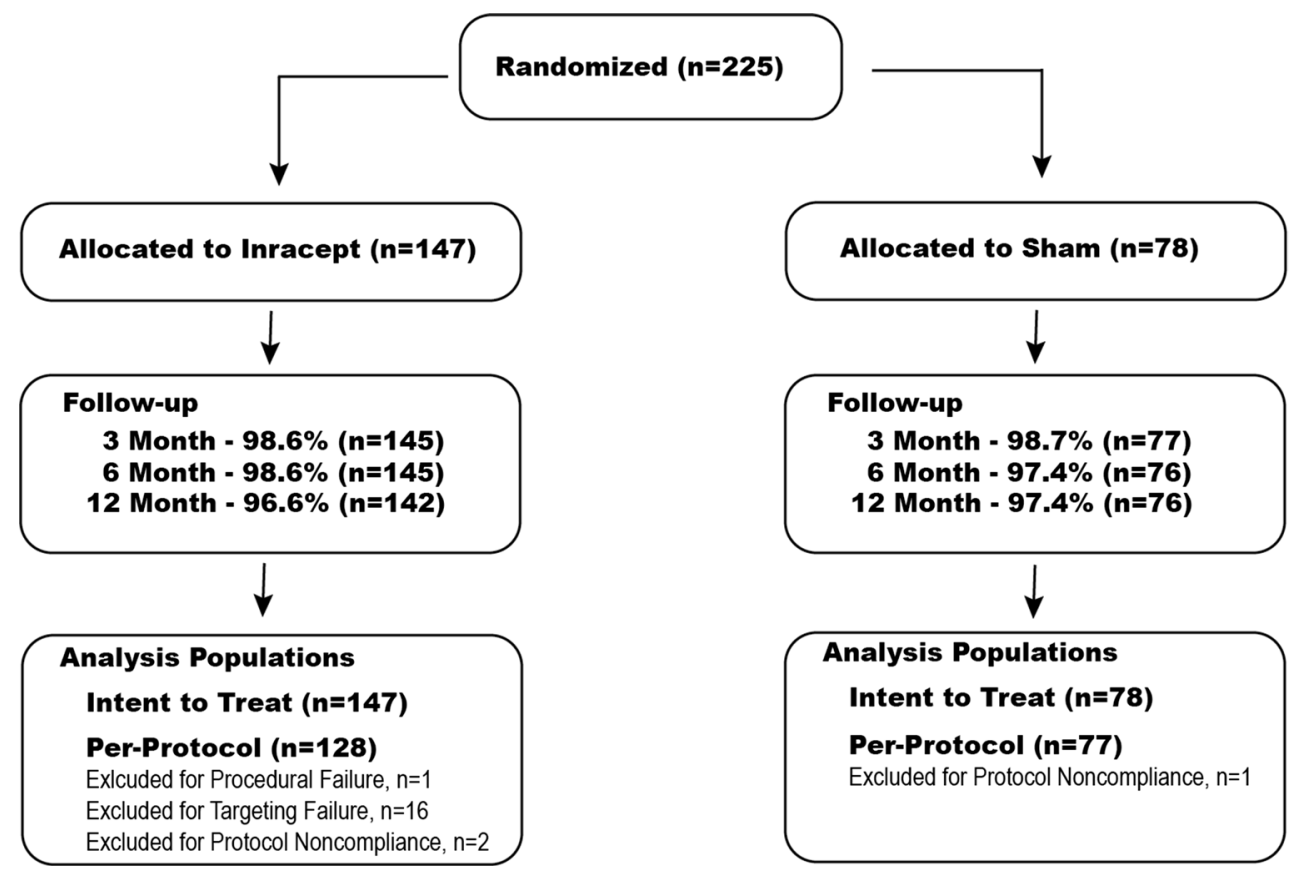

Fig. 2 Flow diagram of the study design 

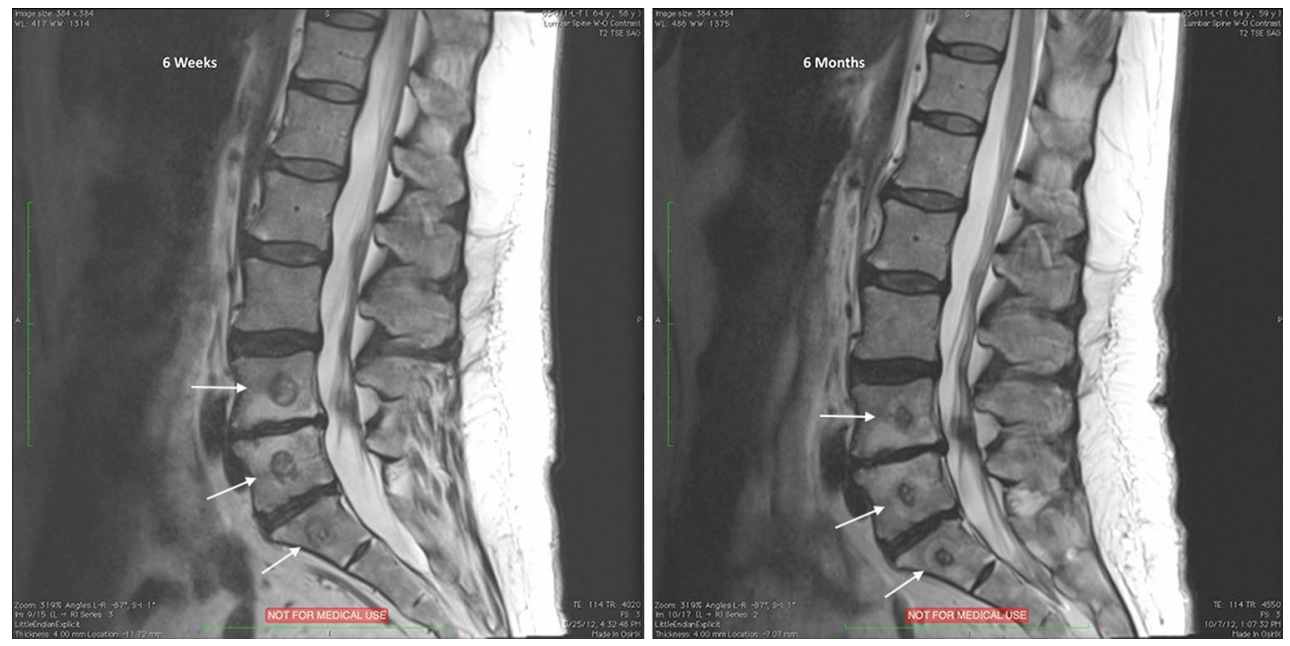

Fig. 3 MR imaging of patient treated and L4-L5-S1 as seen at 6 weeks (left image) and 6 months (right image). The lesion is roughly centered in the middle of the vertebral body; bone remodeling and healing is observed by 6 months

Table 2 Summary of ODI primary end point analyses

\begin{tabular}{|c|c|c|c|}
\hline ITT population & Intracept system arm $(n=147)$ & Sham control $\operatorname{arm}(n=78)$ & $p$ \\
\hline LS mean ODI change from baseline $95 \%$ confidence interval for LS mean & $-19.0[-21.6,-16.5]$ & $-15.4[-18.9,-11.9]$ & 0.107 \\
\hline PP population & Intracept system arm $(n=128)$ & Sham control arm $(n=77)$ & $p$ \\
\hline LS mean ODI change from baseline $95 \%$ confidence interval for LS mean & $-20.5[-23.2,-17.8]$ & $-15.2[-18.7,-11.7]$ & 0.019 \\
\hline
\end{tabular}

( $p=0.083,0.008$, and 0.038 ). The MCID for VAS is $1.5 \mathrm{~cm}$ [14]. Table 3 summarizes the patient reported outcomes for ODI and VAS by follow-up interval and treatment arm; mean values are plotted in Fig. 4.

\section{SF-36, PCS and MCS}

The LSM change in PCS in the PP treatment arm was 9.74, 10.2, and 9.17 at 3, 6, and 12 months, respectively. The LSM change in PCS in the PP sham arm was 9.05, 8.73, and 7.63 at 3, 6, and 12 months, respectively (N.S. all time periods). The MCID for the PCS has been reported as 4.9 [16].

The LSM change in MCS in the PP treatment arm was $2.24,1.90$, and 1.13 at 3,6 , and 12 months, respectively. The LSM change in MCS in the PP sham arm was $0.78,1.17$, and -1.46 at 3,6 , and 12 months, respectively (N.S. 3 and 6 months; $p=0.021$ at 12 months).

\section{Cross-over}

Patients in the sham arm were offered active treatment at 1 year post-op; 57 of 78 patients ( $73 \%$ ) elected to cross-over. Only safety data up to 3 months were collected following the cross-over.

\section{Adverse events}

Adverse events (AEs) were continuously assessed during the study and reported as they occurred; there was no requirement that an event be associated with the study or study device. Each $\mathrm{AE}$ was assessed by the study investigator for severity and relationship to the study device or procedure. An independent review of clinical trial execution and safety data was performed on a regular basis by an external Data Safety Monitoring Board (DSMB) throughout the course of the trial.

There were no device- or procedure-related patient deaths, no unanticipated adverse device effects, and no device-related serious adverse events (SAEs). One devicerelated $\mathrm{AE}$ occurred in a sham patient, who crossed over to the active treatment at 1 year. The patient was on hormone therapy and developed a VCF. Further diagnostic workup revealed concomitant osteopenia. By 8 weeks, the fracture was healed with no further complications.

Eight procedure-related events were reported in six patients following the 225 index procedures, for a complication rate of $2.7 \%$. Two of these six patients were in the sham arm. The events included nerve root injury $(n=1)$, lumbar radiculopathy $(n=2)$, retroperitoneal hemorrhage $(n=1)$, and transient motor or sensory deficits $(n=4)$. The 
Table 3 Summary of ODI and VAS scores (PP population)

\begin{tabular}{|c|c|c|c|c|}
\hline Follow-up period & Variable & $\begin{array}{l}\text { Intracept } \\
\text { system arm } \\
(n=128) \\
\text { Mean (SD) }\end{array}$ & Sham control arm $(n=77)$ & $p^{*}$ \\
\hline \multicolumn{5}{|l|}{ ODI } \\
\hline Baseline & ODI score & $42.4(10.92)$ & $41.2(10.38)$ & \\
\hline 3 months & $\begin{array}{l}\text { ODI score } \\
\text { ODI improvement from baseline }\end{array}$ & $\begin{array}{r}22.1(15.39) \\
-20.3(15.56)\end{array}$ & $\begin{array}{r}25.8(17.44) \\
-15.5(17.87)\end{array}$ & 0.019 \\
\hline 6 months & $\begin{array}{l}\text { ODI score } \\
\text { ODI improvement from baseline }\end{array}$ & $\begin{array}{r}21.6(14.92) \\
-20.8(15.92)\end{array}$ & $\begin{array}{r}25.1(15.29) \\
-16.1(16.38)\end{array}$ & 0.078 \\
\hline 12 months & $\begin{array}{l}\text { ODI score } \\
\text { ODI improvement from baseline }\end{array}$ & $\begin{array}{r}22.6(15.71) \\
-19.8(16.18)\end{array}$ & $\begin{array}{r}25.3(14.92) \\
-15.9(16.20)\end{array}$ & 0.153 \\
\hline \multicolumn{5}{|l|}{ VAS } \\
\hline Baseline & VAS score & $6.73(1.38)$ & $6.64(1.34)$ & \\
\hline 3 months & $\begin{array}{l}\text { VAS score } \\
\text { VAS improvement from baseline }\end{array}$ & $\begin{array}{r}3.80(2.63) \\
-2.90(2.64)\end{array}$ & $\begin{array}{r}4.14(2.64) \\
-2.47(2.49)\end{array}$ & 0.083 \\
\hline 6 months & $\begin{array}{l}\text { VAS score } \\
\text { VAS improvement from baseline }\end{array}$ & $\begin{array}{r}3.74(2.68) \\
-2.98(2.64)\end{array}$ & $\begin{array}{r}4.41(2.76) \\
-2.21(2.45)\end{array}$ & 0.008 \\
\hline 12 months & $\begin{array}{l}\text { VAS score } \\
\text { VAS improvement from baseline }\end{array}$ & $\begin{array}{r}3.96(2.83) \\
-2.76(2.89)\end{array}$ & $\begin{array}{r}4.46(2.78) \\
-2.16(2.69)\end{array}$ & 0.038 \\
\hline
\end{tabular}

* $p$ values from ANCOVA with factors of treatment group, analysis center and treatment group by analysis center interaction, and a covariate of baseline ODI or VAS score
Fig. 4 Mean values of ODI and VAS plotted for all f/u times through 1 year. ODI improvement in treatment arm statistically significant compared to sham arm at 3 months; VAS improvement statistically significant at 6 and 12 months $(p<0.05)$
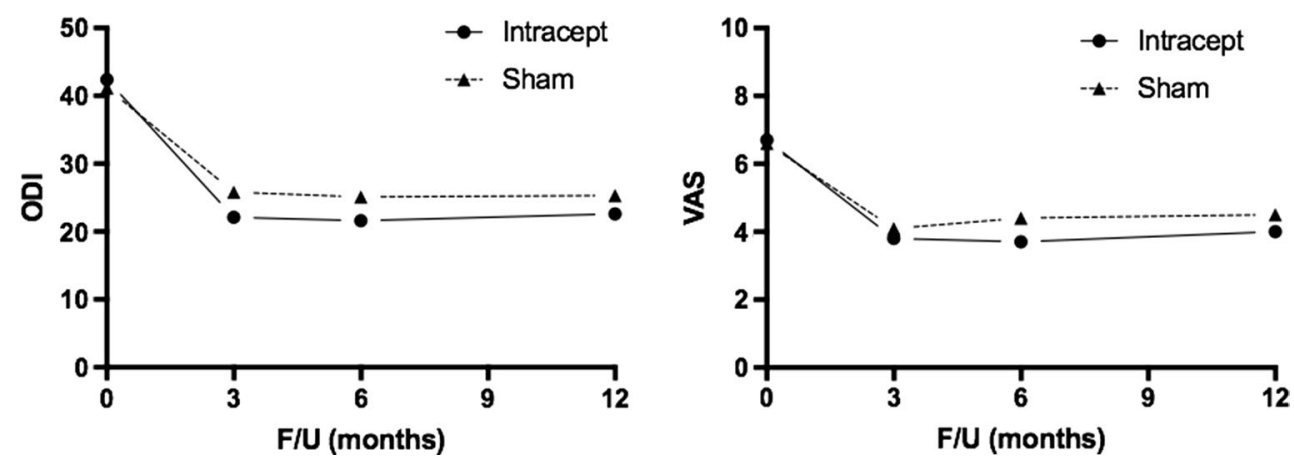

singular case of nerve root injury was in a sham patient, secondary to trocar misplacement during attempted docking at the pedicle. An excessively lateral position resulted in a psoas hematoma, and the resulting compressive lesion caused a transient neuropraxia on the femoral nerve. These events occurred during the perioperative period and the rate between arms was statistically compared using Fisher's Exact test, which determined no significant difference between the arms $(p=1.000)$. All events resolved. There were no reports of aberrant burns, thermal nerve injuries, nor neurological injuries related to the delivery of RF energy within the vertebral body.

MRI evaluation at the 6-week and 6-month follow-up time points found no evidence of any spinal cord abnormalities, avascular necrosis or accelerated disc degeneration. Review of MR imaging at 6-week and 6-month follow-up revealed that a single patient had a change in Modic Type from 1 to 2 between the 6-week and 6-month time points at the inferior level of L5 and the superior level of S1. No patient developed Modic changes at an L3-S1 endplate by 6-month follow-up that was not present at the time of enrollment.

\section{Discussion}

The mechanisms and pathways on the origin of chronic low back pain associated with degenerative changes in the intervertebral disc have been studied for more than half a century. Unlike etiologies with a clear pathological cause, such as disc herniation, spondylolisthesis or spinal stenosis, CLBP appears to arise in part following disc and endplate degeneration with subsequent changes in the endplate morphology and biology. Inflammatory mediators have been 
shown to be expressed following disc injury, leading to a cascade of further inflammatory response via cytokine production [17].

Direct evidence supporting the vertebral body as a source of clinical pain was provided by Kuslich et al. who reported on a series of patients during laminectomy using local anesthesia only. They reported that direct intraoperative mechanical stimulation of the endplates of these awake patients consistently provoked significant pain response [18]. Additional evidence for the endplate's role in generation of pain signals was provided by Lotz et al., who performed a histomorphological study of human vertebral bodies which documented endplate nociceptor densification with increased disc degeneration [19].

Heggeness et al. observed that injection into the disc, as during discography, caused the endplates to deform and hypothesized that this deformation could account for the pain experienced during a discogram in some patients [20]. After hypothesizing that increased physical activity could instigate disc degeneration, Adams et al. showed that minor damage to the vertebral endplates could lead to structural changes in the adjacent intervertebral discs [21, 22]. Carragee et al. determined that vertebral body and endplate MRI signal changes, indicative of intraosseous edema or inflammation, were well correlated with clinical low back pain [23].

Further correlation between vertebral body pathology and CLBP was described by Modic et al. who described intraosseus MRI changes adjacent to the vertebral endplates in patients with CLBP [24]. Weishaupt et al. reported $100 \%$ specificity to pain in patients with Type 1 and Type 2 Modic changes and furthermore observed strong and very similar positive predictive values and specificity associated with both Type 1 (88\%) and Type 2 (96\%) Modic changes [25]. Kuisma et al. found a 2.28 odds ratio for the presence of Modic changes at L5-S1 in patients with CLBP [26]. Although Rahme and Mossa found that low back pain is more commonly associated with Type I Modic changes, they also noted that this association was sometimes made based on relatively small sample sizes, and that other studies showed correlation between both Type 1 and Type 2 Modic changes and low back pain [27]. Schroeder et al. recently observed cytokine elevation in patients with Modic Type 2 MR signals treated with anterior lumbar fusion, indicating that both Type 1 and Type 2 changes may be associated with low back pain [28].

In longitudinal studies, a change in Modic presentation from Type 1 to Type 2 has been observed, with the concomitant observation that pain was more likely associate with Type 1 presentation [24, 29]. In the present study, approximately $68 \%$ of patients reported experiencing low back pain for more than 5 years, suggesting that the higher baseline incidence of Modic Type 2 changes observed may reflect the transition of Modic Type 1 findings to Type 2 over time. Kjaer et al. have observed that Type 1 Modic changes are correlated with more recent low back pain, which may in part explain the higher prevalence of Type 2 findings in the SMART patient population who were symptomatic for over half a decade [30,31]. Note that during the course of the SMART study itself, only a single instance of Type 1 to Type 2 conversion was observed. No statistical difference was observed in outcome between patients presenting with Type 1 versus Type 2 Modic changes.

The SMART trial was designed to test the hypothesis that the BVN plays an important role in the transmission of pain signals in patients with CLBP. The data showed that ablation of the BVN in the treatment arm decreased patients' mean ODI by more than twice the MCID. The magnitude of the decrease was in line with decreases in ODI previously observed following fusion and total disc replacement (TDR) surgery. Two common TDRs, the Charité and the ProDisc, report an average percent improvement in ODI of $42.0 \%$ at 3 months, which compares favorably with the $48.5 \%$ improvement at 3 months observed in the treatment arm of the present study $[32,33]$. These TDR studies were controlled against fusion; the average percent improvement in ODI of the patients receiving fusion was 35.0\%. However, the patients in these TDR studies were permitted to enroll with conditions other than just isolated back pain, including stenosis or disc herniation. Studies looking at strictly chronic low back patients report lower percentage improvements in ODI. For example, Fritzell et al. reported a $25 \%$ improvement in ODI in their CLBP patients surgically treated with fusion, whereas Brox et al. reported a 37\% improvement in ODI in fused CLBP patients [1,34]. Thus, the improvement in patient outcome observed in the treatment arm of the SMART study was comparable to that observed following TDR and fusion procedures for similar etiologies.

The mean improvement in ODI in the ITT sham arm was 15.4 points at 3 months, and was durable at 6 and 12 months. Positive responses to placebo or sham treatments are well-recognized, especially in chronic pain populations, where the degree and duration of sham effectiveness often approaches that of active treatment [35-38]. Furthermore, multiple studies evaluating medical devices against sham interventions also suggest a correlation between the magnitude of the sham response and the invasiveness of the treatment [39-45].

The cognitive perception of chronic pain is a function of peripheral nociceptor input and complex central neurobiological modulation. Historically, clinical placebo or sham effects were attributed to statistical and not biological factors. Placebos were included in trials to statistically filter out the "noise" not associated with the mechanism of action of the proposed pharmacological or surgical treatment itself. In such a model, the total treatment effect is 
a linear superposition of the placebo effect and the interventional effect. A high placebo response in such a linear model is interpreted to indicate that the treatment effect is minimal and the procedure or drug is not particularly effective. However, newer models, particularly when studying pain, recognize that modulation of the central brain response through modification of expectations is an integral part of the patient's response to any treatment. These models incorporate the sham response as part of the overall treatment effect, and have been developed to the point where they can distinguish which patients are going to have a higher central brain response (sham response) as opposed to peripheral response (intervention response).

Supporting this neurobiological basis for a placebo or sham response, Tétreault et al. performed an elegant study where they first confirmed brain activity associated with a placebo response, and then a priori predicted which patients in a subsequent study would show a placebo response [46]. The ability to predict, opposed to just document, a placebo response and to map areas of the brain associated with that response suggests that in a given population there exist a range of patient responses to a specific pain alleviation therapy, from almost pure central brain placebo response to nearly pure peripheral stimulatory inhibitory response. Treatments may act on one or the other response in varying degrees, but the documentation through randomized controlled study of the ability to relieve both peripheral and central pain is important to the adoption of a new surgical therapy. The ability in the SMART trial to distinguish the active treatment from the sham treatment suggests that ablation of the BVN has therapeutic value, although the overall pain response in a given patient is a complex function of the combined effects of placebo and treatment.

Radiofrequency ablation of the BVN for the relief of chronic low back pain represents a new treatment modality and is distinct from other RF ablation therapies used in the spine such as facet ablation. BVN ablation is technically and scientifically distinct from facet ablation because it uses a transpedicular approach into the vertebral body to access the BVN directly to alleviate vertebrogenic pain, and furthermore mandates strict patient selection criteria. Note that facet ablation may have varying effectiveness as a function of patient selection, as recently reported by Juch et al. [47].

In the present study, using a 10-point ODI improvement as a threshold, $75.6 \%$ of treatment arm patients as opposed to $55.3 \%$ of sham arm patients were characterized as responders. In addition, the treatment group reported statistically significant improved outcomes compared to the sham group. Comparison of the difference in outcome score between the sham and treatment groups does not represent the clinical utility of the Intracept Procedure because a sham treatment is not a clinically acceptable treatment for CLBP, nor is a sham response likely to occur in an open label setting. The overall therapeutic value of the procedure should be viewed through its safety profile and observed improvements from patient baseline, which are the same filters applied to other more invasive procedures which have not been compared to sham treatment. The results of this study support BVN ablation as a minimally invasive treatment for relief of chronic low back pain.

\section{Summary}

We report on a multi-center, randomized, double-blind, sham-controlled trial conducted on a well-defined cohort of patients with chronic low back pain presenting with concomitant Modic changes at the diagnosed level. Patients in the per-protocol analysis treated using RF ablation of the BVN nerve complex exhibited statistically superior relief of disability and a higher response rate than those treated with the sham procedure.

Acknowledgements The authors thank all of the physicians who participated in the treatment and follow-up of the patients in the SMART study.

\section{Compliance with ethical standards}

Conflict of interest None of the authors has any potential conflict of interest.

Open Access This article is distributed under the terms of the Creative Commons Attribution 4.0 International License (http://creativeco mmons.org/licenses/by/4.0/), which permits unrestricted use, distribution, and reproduction in any medium, provided you give appropriate credit to the original author(s) and the source, provide a link to the Creative Commons license, and indicate if changes were made.

\section{References}

1. Fritzell P, Hagg O, Wessberg P, Nordwall A (2001) 2001 Volvo Award Winner in Clinical Studies: lumbar fusion versus nonsurgical treatment for chronic low back pain: a multicenter randomized controlled trial from the Swedish Lumbar Spine Study Group. Spine 26(23):2521-2532 (discussion 2532-2534)

2. Brox JI, Reikeras O, Nygaard O, Sorensen R, Indahl A, Holm I, Keller A, Ingebrigtsen T, Grundnes O, Lange JE, Friis A (2006) Lumbar instrumented fusion compared with cognitive intervention and exercises in patients with chronic back pain after previous surgery for disc herniation: a prospective randomized controlled study. Pain 122(1-2):145-155. https://doi.org/10.1016/j. pain.2006.01.027

3. Guyer RD, Pettine K, Roh JS, Dimmig TA, Coric D, McAfee PC, Ohnmeiss DD (2014) Comparison of 2 lumbar total disc replacements: results of a prospective, randomized, controlled, multicenter Food and Drug Administration trial with 24-month follow-up. Spine 39(12):925-931. https://doi.org/10.1097/brs.00000 00000000319 
4. Delamarter R (2011) Prospective, randomized, multicenter food and drug administration investigational device exemption study of the ProDisc-L total disc replacement compared with circumferential arthrodesis for the treatment of two-level lumbar degenerative disc disease: results at twenty-four months. J Bone Jt Surg (American) 93(8):705. https://doi.org/10.2106/jbjs.i.00680

5. Bogduk N, Tynan W, Wilson AS (1981) The nerve supply to the human lumbar intervertebral discs. J Anat 132(Pt 1):39-56

6. Sherman MS (1963) The nerves of bone. J Bone Jt Surg 45(3):522-528

7. Crock HV, Yoshizawa H (1976) The blood supply of the lumbar vertebral column. Clin Orthop Relat Res 115:6-21

8. Brown MF, Hukkanen MV, McCarthy ID, Redfern DR, Batten JJ, Crock HV, Hughes SP, Polak JM (1997) Sensory and sympathetic innervation of the vertebral endplate in patients with degenerative disc disease. J Bone Jt Surg Br 79(1):147-153

9. Antonacci MD, Mody DR, Heggeness MH (1998) Innervation of the human vertebral body: a histologic study. J Spinal Disord 11(6):526-531

10. Bailey JF, Liebenberg E, Degmetich S, Lotz JC (2011) Innervation patterns of PGP 9.5-positive nerve fibers within the human lumbar vertebra. J Anat 218(3):263-270. https://doi.org/10.111 $1 /$ j.1469-7580.2010.01332.x

11. Fras C, Kravetz P, Mody DR, Heggeness MH (2003) Substance P-containing nerves within the human vertebral body. An immunohistochemical study of the basivertebral nerve. Spine J $3(1): 63-67$

12. Fagan A, Moore R, Vernon Roberts B, Blumbergs P, Fraser R (2003) ISSLS prize winner: the innervation of the intervertebral disc: a quantitative analysis. Spine 28(23):2570-2576. https:// doi.org/10.1097/01.brs.0000096942.29660.b1

13. Becker S, Hadjipavlou A, Heggeness MH (2017) Ablation of the basivertebral nerve for treatment of back pain: a clinical study. Spine J 17(2):218-223. https://doi.org/10.1016/j.spine e.2016.08.032

14. Ostelo RW, de Vet HC (2005) Clinically important outcomes in low back pain. Best Pract Res Clin Rheumatol 19(4):593-607. https://doi.org/10.1016/j.berh.2005.03.003

15. Hagg O, Fritzell P, Nordwall A (2003) The clinical importance of changes in outcome scores after treatment for chronic low back pain. Eur Spine J 12(1):12-20. https://doi.org/10.1007/ s00586-002-0464-0

16. Copay AG, Glassman SD, Subach BR, Berven S, Schuler TC, Carreon LY (2008) Minimum clinically important difference in lumbar spine surgery patients: a choice of methods using the Oswestry Disability Index, Medical Outcomes Study questionnaire Short Form 36, and pain scales. Spine J 8(6):968-974. https://doi.org/10.1016/j.spinee.2007.11.006

17. Ulrich JA, Liebenberg EC, Thuillier DU, Lotz JC (2007) ISSLS prize winner: repeated disc injury causes persistent inflammation. Spine 32(25):2812-2819. https://doi.org/10.1097/ BRS.0b013e31815b9850

18. Kuslich SD, Ulstrom CL, Michael CJ (1991) The tissue origin of low back pain and sciatica: a report of pain response to tissue stimulation during operations on the lumbar spine using local anesthesia. Orthop Clin N Am 22(2):181-187

19. Lotz JC, Fields AJ, Liebenberg EC (2013) The role of the vertebral end plate in low back pain. Glob Spine $\mathrm{J}$ 03(EFirst):153-164. https://doi.org/10.1055/s-0033-1347298

20. Heggeness MH, Doherty BJ (1993) Discography causes end plate deflection. Spine 18(8):1050-1053

21. Adams MA, Dolan P (1997) Could sudden increases in physical activity cause degeneration of intervertebral discs? Lancet 350(9079):734-735. https://doi.org/10.1016/s0140 $-6736(97) 03021-3$
22. Adams MA, Freeman BJ, Morrison HP, Nelson IW, Dolan P (2000) Mechanical initiation of intervertebral disc degeneration. Spine 25(13):1625-1636

23. Carragee EJ, Alamin TF, Miller JL, Carragee JM (2005) Discographic, MRI and psychosocial determinants of low back pain disability and remission: a prospective study in subjects with benign persistent back pain. Spine J 5(1):24-35. https://doi. org/10.1016/j.spinee.2004.05.250

24. Modic MT, Steinberg PM, Ross JS, Masaryk TJ, Carter JR (1988) Degenerative disk disease: assessment of changes in vertebral body marrow with MR imaging. Radiology 166(1 Pt 1):193-199

25. Weishaupt D, Zanetti M, Hodler J, Min K, Fuchs B, Pfirrmann CWA, Boos N (2001) Painful lumbar disk derangement: relevance of endplate abnormalities at MR imaging. Radiology 218(2):420-427

26. Kuisma M, Karppinen J, Niinimaki J, Ojala R, Haapea M, Heliovaara M, Korpelainen R, Taimela S, Natri A, Tervonen O (2007) Modic changes in endplates of lumbar vertebral bodies: prevalence and association with low back and sciatic pain among middle-aged male workers. Spine 32(10):1116-1122. https://doi. org/10.1097/01.brs.0000261561.12944.ff

27. Rahme R, Moussa R (2008) The modic vertebral endplate and marrow changes: pathologic significance and relation to low back pain and segmental instability of the lumbar spine. AJNR Am J Neuroradiol 29(5):838-842. https://doi.org/10.3174/ajnr.A0925

28. Schroeder GD, Markova DZ, Koerner JD, Rihn JA, Hilibrand AS, Vaccaro AR, Anderson DG, Kepler CK (2017) Are Modic changes associated with intervertebral disc cytokine profiles? Spine J 17(1):129-134. https://doi.org/10.1016/j.spinee.2016.08.006

29. Mitra D, Cassar-Pullicino VN, McCall IW (2004) Longitudinal study of vertebral type-1 end-plate changes on MR of the lumbar spine. Eur Radiol 14(9):1574-1581. https://doi.org/10.1007/s0033 0-004-2314-4

30. Kjaer P, Korsholm L, Bendix T, Sorensen JS, Leboeuf-Yde C (2006) Modic changes and their associations with clinical findings. Eur Spine J 15(9):1312-1319. https://doi.org/10.1007/s0058 6-006-0185-x

31. Kjaer P, Leboeuf-Yde C, Korsholm L, Sorensen JS, Bendix T (2005) Magnetic resonance imaging and low back pain in adults: a diagnostic imaging study of 40 -year-old men and women. Spine 30(10):1173-1180

32. Blumenthal S, McAfee PC, Guyer RD, Hochschuler SH, Geisler FH, Holt RT, Garcia R Jr, Regan JJ, Ohnmeiss DD (2005) A prospective, randomized, multicenter Food and Drug Administration investigational device exemptions study of lumbar total disc replacement with the CHARITE artificial disc versus lumbar fusion: part I: evaluation of clinical outcomes. Spine 30(14):15651575 (discussion E1387-E1591)

33. Zigler J, Delamarter R, Spivak JM, Linovitz RJ, Danielson GO 3rd, Haider TT, Cammisa F, Zuchermann J, Balderston R, Kitchel S, Foley K, Watkins R, Bradford D, Yue J, Yuan H, Herkowitz H, Geiger D, Bendo J, Peppers T, Sachs B, Girardi F, Kropf M, Goldstein J (2007) Results of the prospective, randomized, multicenter Food and Drug Administration investigational device exemption study of the ProDisc-L total disc replacement versus circumferential fusion for the treatment of 1-level degenerative disc disease. Spine 32(11):1155-1162. https://doi.org/10.1097/brs.0b013e3180 54e 377 (discussion 1163)

34. Brox JI, Sorensen R, Friis A, Nygaard O, Indahl A, Keller A, Ingebrigtsen T, Eriksen HR, Holm I, Koller AK, Riise R, Reikeras O (2003) Randomized clinical trial of lumbar instrumented fusion and cognitive intervention and exercises in patients with chronic low back pain and disc degeneration. Spine 28(17):1913-1921. https://doi.org/10.1097/01.brs.0000083234.62751.7a

35. Benedetti F (2008) Mechanisms of placebo and placebo-related effects across diseases and treatments. Annu Rev Pharmacol 
Toxicol 48:33-60. https://doi.org/10.1146/annurev.pharm tox.48.113006.094711

36. Chappell AS, Desaiah D, Liu-Seifert H, Zhang S, Skljarevski V, Belenkov Y, Brown JP (2011) A double-blind, randomized, placebo-controlled study of the efficacy and safety of duloxetine for the treatment of chronic pain due to osteoarthritis of the knee. Pain Pract 11(1):33-41. https://doi.org/10.111 1/j.1533-2500.2010.00401.x

37. Moore RA, Moore OA, Derry S, Peloso PM, Gammaitoni AR, Wang H (2010) Responder analysis for pain relief and numbers needed to treat in a meta-analysis of etoricoxib osteoarthritis trials: bridging a gap between clinical trials and clinical practice. Ann Rheum Dis 69(2):374-379. https://doi.org/10.1136/ ard.2009.107805

38. Freynhagen R, Strojek K, Griesing T, Whalen E, Balkenohl M (2005) Efficacy of pregabalin in neuropathic pain evaluated in a 12-week, randomised, double-blind, multicentre, placebo-controlled trial of flexible- and fixed-dose regimens. Pain 115(3):254 263. https://doi.org/10.1016/j.pain.2005.02.032

39. Moseley JB Jr, Wray NP, Kuykendall D, Willis K, Landon G (1996) Arthroscopic treatment of osteoarthritis of the knee: a prospective, randomized, placebo-controlled trial. Results of a pilot study. Am J Sports Med 24(1):28-34

40. O'Rourke DA, O'Rourke HM (1964) Removal of the carotid body for asthma: an appraisal of results. Med J Aust 2:869-870

41. Dimond EG, Kittle CF, Crockett JE (1960) Comparison of internal mammary artery ligation and sham operation for angina pectoris. Am J Cardiol 5:483-486
42. Cobb LA, Thomas GI, Dillard DH, Merendino KA, Bruce RA (1959) An evaluation of internal-mammary-artery ligation by a double-blind technic. N Engl J Med 260(22):1115-1118. https:// doi.org/10.1056/nejm195905282602204

43. Deyo RA, Walsh NE, Martin DC, Schoenfeld LS, Ramamurthy S (1990) A controlled trial of transcutaneous electrical nerve stimulation (TENS) and exercise for chronic low back pain. N Engl J Med 322(23):1627-1634. https://doi.org/10.1056/nejm199006 073222303

44. Langley GB, Sheppeard H, Johnson M, Wigley RD (1984) The analgesic effects of transcutaneous electrical nerve stimulation and placebo in chronic pain patients. A double-blind non-crossover comparison. Rheumatol Int 4(3):119-123

45. Kaptchuk TJ, Goldman P, Stone DA, Stason WB (2000) Do medical devices have enhanced placebo effects? J Clin Epidemiol 53(8):786-792

46. Tetreault P, Mansour A, Vachon-Presseau E, Schnitzer TJ, Apkarian AV, Baliki MN (2016) Brain connectivity predicts placebo response across chronic pain clinical trials. PLoS Biol 14(10):e1002570. https://doi.org/10.1371/journal.pbio.1002570

47. Juch JNS, Maas ET, Ostelo R, Groeneweg JG, Kallewaard JW, Koes BW, Verhagen AP, van Dongen JM, Huygen F, van Tulder MW (2017) Effect of radiofrequency denervation on pain intensity among patients with chronic low back pain: the mint randomized clinical trials. JAMA J Am Med Assoc 318(1):68-81. https://doi. org/10.1001/jama.2017.7918

\section{Affiliations}

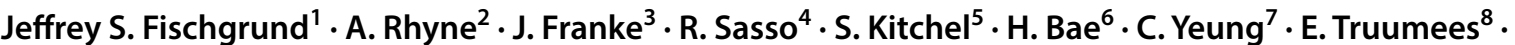

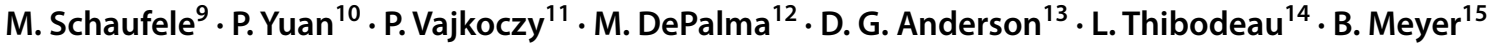

Jeffrey S. Fischgrund

jsfischgrund37@gmail.com

1 Department of Orthopedic Surgery, Oakland University William Beaumont School of Medicine, 3535 West 13 Mile Road, Suite 744, Royal Oak, MI 48073, USA

2 Ortho Carolina Spine Center, Charlotte, USA

3 Department of Orthopedics-Spine and Pediatric Orthopedics, Klinikum Magdeburg GmbH, Magdeburg, Germany

4 Department of Orthopedic Surgery, Indiana University School of Medicine, Indianapolis, USA

5 NeuroSpine Institute, Eugene, USA

6 Department of Surgery, Cedars Sinai Medical Center, Los Angeles, USA

7 Desert Institute for Spine Care, Phoenix, USA
8 Seton Spine and Scoliosis Center, Seton Medical Center, Brackenridge University Hospital, Austin, USA

9 Pain Solutions Treatment Centers, Marietta, USA

10 Long Beach Memorial Medical Center, Long Beach, USA

11 Department of Neurosurgery, Charité-Universitaetsmedizin Berlin, Campus Virchow Medical Center, Berlin, Germany

12 Virginia iSpine Physicians, Richmond, USA

13 Departments of Orthopaedic and Neurological Surgery, Thomas Jefferson University, Philadelphia, USA

14 Maine Spine Surgery, Portland, USA

15

Direktor der Neurochirurgischen Klinik und Poliklinik, Klinikum Rechts der Isar, Munich, Germany 